\title{
MODULAR DESIGNED, WHEELCHAIR BASED ORTHOTIC SYSTEM FOR UPPER EXTREMITIES
}

\author{
By Peter H. Stern, M.D. \\ Spinal Cord Injury Unit, The Burke Rehabilitation Centre, White Plains, \\ New York 10605 \\ and \\ Thomas LAUKo \\ Bioengineering, Orthotics and Prosthetics, The Burke Rehabilitation Centre, \\ White Plains, New York 10605
}

\section{INTRODUCTION}

THE design fabrication and utilisation of orthotic systems for flail arms and hands has not kept pace with the dramatic developments in orthopedic technology over the last decade. Koch (1974) considers orthotic aids to the disabled a 'stepchild' of orthopaedics, particularly when compared with the advances made in the application of internal and external prostheses. Yet the demand for useful and cost-effective devices is increasing as a result of mounting incidence of spinal cord injury (SCI) with a marked shift of the level of injuries from para to quadriplegia.

This presentation is limited to wheelchair based, hand/arm assistive devices for patients with severe upper extremity dysfunction such as encountered in SCI level above C5. The basic principle of all devices currently employed is that of an exoskeleton which harnesses the paralysed hand and arm and which a patient can use as a type of manipulator. Industrial production of thermo-plastic materials, miniaturised power sources and electronic controls have provided new impetus for advancing both the science and art in upper extremity orthotics; hopefully, to approximate the achievements in upper limb prosthetics.

\section{GENERAL DEVICE DESCRIPTION AND TERMINOLOGY}

Before prescription principles can be discussed, it is necessary to clarify some of the almost arcane terminology of orthotic devices and their components. To do this, it is advantageous to use terminology analogous to that used in prosthetics; in as much as there is a current tendency to employ prosthetic principles in orthotics as well. Basic orthotic components ranging from distal to proximal parts of the upper extremities are:

I. Terminal Devices. These are usually three or two jaw chuck devices which provide thumb and finger prehension, either wrist driven (tenodesis) or externally powered. Its prosthetic counterpart is the functional hand or hook.

2. Wrist Units. These are usually supportive or stabilising structures but the capability of pro and supination can be provided. Its prosthetic counterpart is the externally powered I.N.A.I.L. wrist unit.

I2/4-U 

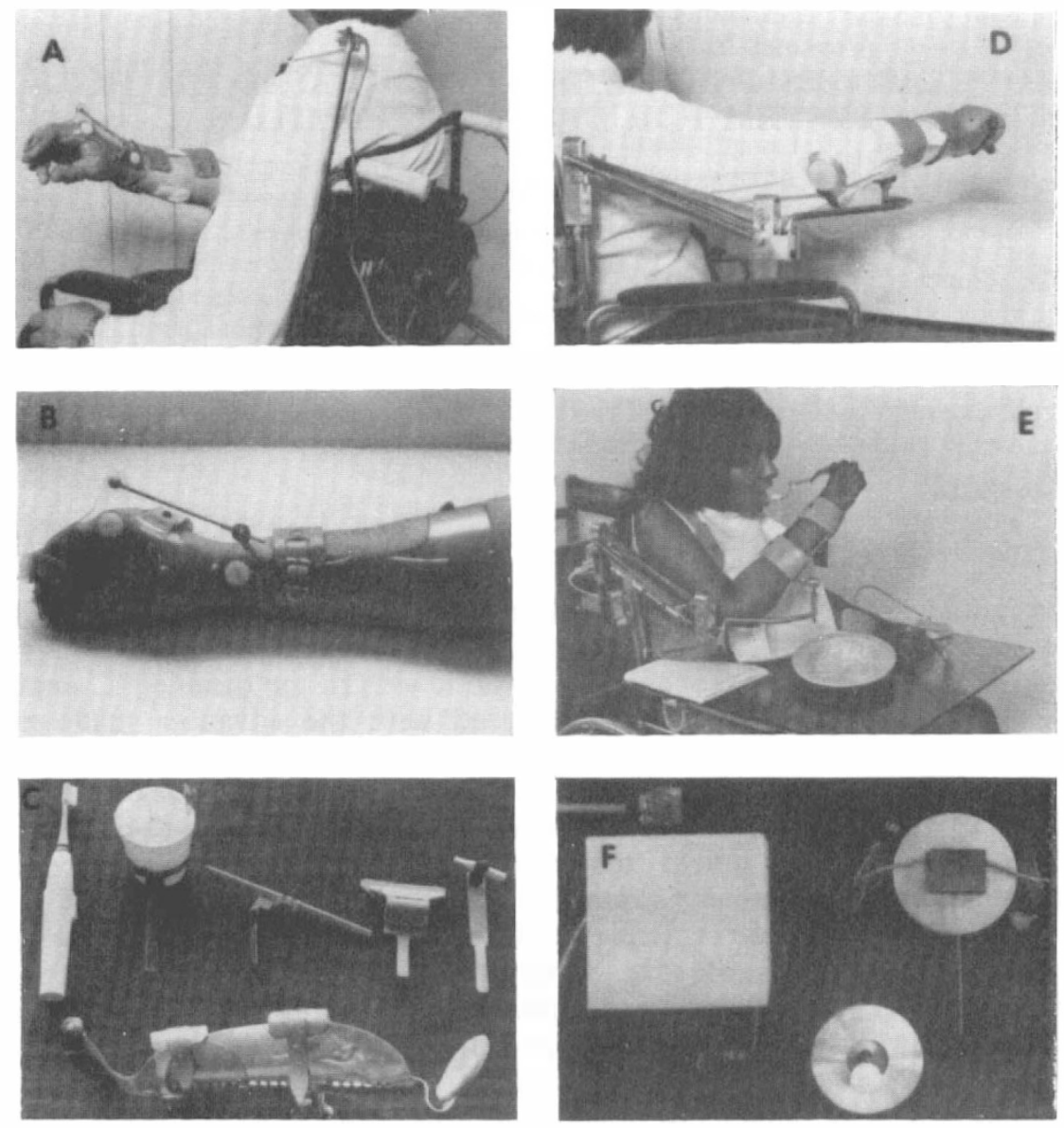

FIG. I

A. Electrically driven prehension orthosis with unidirectional shoulder control. B. Wrist driven prehension orthosis. C. Forearm and static wrist extension module with universal receptacle and various attachments. D. Spring pre-loaded, four-bar linkage module. E. Electrically driven, four-bar linkage and forearm modules in use. F. Clockwise: key control, bi-directional lever control, joy-stick control.

3. Forearm Support. The positioning of this supportive structure; i.e. volar, dorsal or lateral, depends on the type of terminal or elbow unit required. Its prosthetic counterpart is the forearm shell.

4. Elbow Unit. This is usually a hinged type device but unlike its prosthetic counterpart, external power has to be remote.

5. Arm Support. Its configuration and positioning is dependent on both the need for an elbow and/or shoulder unit. It corresponds to the arm shell in upper limb prosthetics.

6. Shoulder Unit. This is a complex mechanism for which there is no true prosthetic counterpart except for a passive abductor hinge connecting a shoulder 


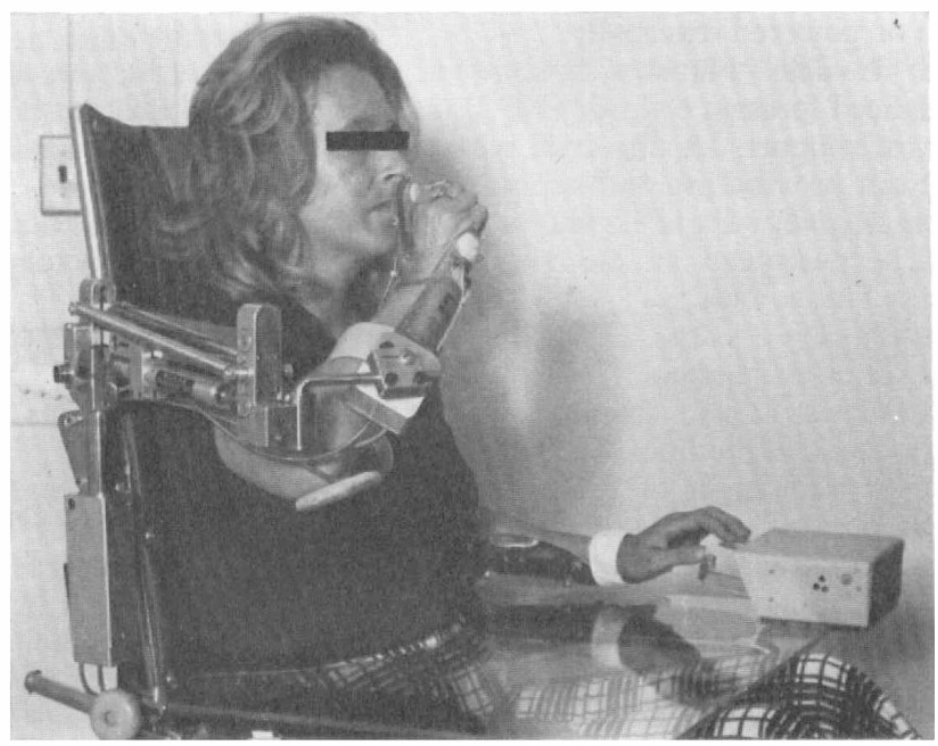

FIG. 2

Electrically driven modular arm orthosis with five degrees of freedom in use.

pad to the arm in cases of shoulder disarticulation. In orthotics a flail shoulder can be assisted by providing externally powered modules for flexion extension, abduction, cross adduction and abduction in the horizontal plane and shoulder rotation. The details of this will be subject to further explanations.

\section{GENERAL PRESCRIPTION PRINCIPLES}

The basic prescription principles used by the Department of Bioengineering, Orthotics and Prosthetics of The Burke Rehabilitation Centre do not differ much from those employed elsewhere. As in any other branch of medicine, a good prescription is based on a thorough diagnostic evaluation. This must include an assessment of joint range of motion, residual muscle power and sensory testing. One must bear in mind that neurological deficits in the upper extremity can present a perplexing picture. Cervical lesions can be incomplete, root escape or recovery is often encountered. Some brachial plexus lesions result in a flail shoulder and arm with retained hand function. Spasticity and contractures are the single most hindering factor for successful orthotic application. Sensory deficits require orthotic design modifications.

\section{DESCRIPTION OF THE BURKE MODULAR ORTHOTIC SYSTEM}

I. Terminal Module. Figure I, A and D shows terminal devices which are made from moulded acrylic nylon. The fabrication of this acrylic nylon structure requires a plaster of paris mould made from the patient's hand and forearm. This, of course, improves the fit of the device, which can be adjusted by simply heating 
the material with a heat gun. The terminal device can be wrist driven by an actuator rod or powered externally.

2. Wrist Module. Figure I, D, shows an acrylic nylon structure attached to a standard balanced forearm orthotic (BFO) through, which in this combination also acts as forearm support. A universal receptacle is provided. An axially actuated spiral sleeve can provide pro and supination.

3. Elbow Module. Figure 2 shows a bi-directionally driven flexion extension module which consists of an electric-motor-driven bevel gear actuator located laterally to the elbow joint.

4. Shoulder/Arm Module. Figure I, D, shows this module which consists of a spring loaded four-bar linkage. The proximal ball-bearing housing attaches to a standard BFO bracket and the distal ball-bearing housing contains the sleeve receptacle for forearm modules.

By proper adjustment of the springs, gravity of the upper extremity can practically be eliminated. Figure 6 shows this module powered by an electric driven ball screw actuator resulting in shoulder flexion up to 90 degrees.

Figure I, E, shows the proximal ball-bearing housing, powered by a directly linked bi-directional motor providing mobility in the horizontal plane.

The described modules can provide a completely powered arm orthosis up to five degrees of freedom (Figure 2). In the presence of residual muscle function the described modules can be combined with swivel arms or spring-assisted modules.

\section{CONTROLS}

Control sites as well as control modules must be carefully selected. The Burke system uses three basic control modules:

I. Foy-stick (fig. I, F). This system was used with good results for the three, four, and five degrees of freedom systems in triangular, quadrilateral and pentagonal switching arrangements.

2. Key Control (fig. I, F). A sensitive key control was used for a patient with minimal residual finger function on the contralateral side. This module controlled a five degrees of freedom system allowing a theoretical combination of 242 motions. However, the patient was not able to master the operation of more than three modules at a time.

3. Micro-switch Assemblies. Several different switch assemblies were used for single joint control. Figure I, A, shows a one-directional shoulder actuated switch. Figure I, E F, shows a bi-directional lap board switch for contralateral hand control.

\section{OPERATING SPECIFICATIONS}

Externally powered upper limb prostheses are generally able to carry out a given joint range in one second. Proportional controls are now replacing digital (on-off) controls.

In orthotic modules, speed control is still in the experimental stages. We found that a speed of a given joint range of motion of two seconds was generally acceptable. The torque provided by the shoulder module was approximately 
I 20 inch/lb. The terminal (prehension) module was adjustable to two lb. pressure between thumb and fingers.

Electrical range of motion limit switches are used and backed by mechanical stops for fail-safe operation.

\section{DISCUSSION}

The Burke modular upper extremity orthotic system is a further development of previously described apparatus such as the IRM electric arm orthosis (Lehneis and Wilson, I972). That system uses remotely placed actuators and Bowden cable power transmission in order to avoid conspicuous appearance and added weight of the orthosis. We did provide a patient, with almost complete flaccid quadriplegia with a modified IRM electric arm orthosis but found the Bowden cable transmission not reliable, cumbersome and actually more conspicuous than direct drive. Consequently, we developed directly driven joints eliminating, whenever possible, Bowden cable power transmission. The direct driven finger prehension module under development is using universal joint linkage rather than a Bowden cable to move the actuator lever.

All sophisticated, externally powered orthotic systems seem to have at least two major problems in common:

I. Controls.

2. Unfavourable cost-benefit ratio.

The Burke system tries to avoid conspicuous if not bizarre control sites such as tongue control, Ranchos (Nickel et al., I969), or myoelectric control using facial muscles (Rehabilitation Institute of Montreal), although it is realised that contralateral control can present considerable problems. Breath, voice, or puff-suck controls appear unsuitable to control an arm orthosis since these controls will interfere with basic self-care function such as the patient's ability to eat and drink. It was also observed that most patients find it difficult to effectively control devices with five degrees of freedom or more. Technically it is possible to computerise a complete set of motions (Langrana, I974) but it has been said that 'patients resent robotisations'.

The unfavourable cost benefit ratio is mainly a result of the high production costs of custom-made devices. Consequently, efforts are being made to go forward with modular designs utilising standard commercially available components whenever possible and eventually mass-produce interchangeable modules as described.

Medical personnel involved in the care of high quadriplegic patients present a wide spectrum of attitudes towards complex orthotic systems. This ranges from therapeutic nihilism and resultant under-prescription, to technological overenthusiams and resultant over-prescription. Patients perceive these attitudes and react to the notion that 'nothing further can be done', or when a complicated, expensive system fails to produce the desired results. It is our opinion that patients have a right to be given a choice of systems ranging from simple to complex. Therefore, it is necessary to provide orthotic departments with a wide selection of orthotic modules which can be 'pulled off the shelf' and applied on a temporary basis for therapeutic trials. This was one of the main objectives of pursuing the modular design concept of the described system, which we hope to develop further. 


\section{SUMMARY}

This is a description of a wheelchair based, modular upper extremity orthotic system for patients with profound paralysis such as encountered in patient with SCI above the $\mathrm{C}_{5}$ level. The need for readily available, pre-fabricated components is stressed. More research is required to optimise orthotic controls.

\section{REFERENCES}

KocH, F. (1974). The application of external power in upper extremity orthotic devices. Orthopaedic Technik, 2.

LANGRANA, N. A. (I974). An automated method of dynamic analysis of spatial linkages: white special applications to biomechanics. Thesis presented to the Faculty of the Graduate School of Cornell University of the Degree of Doctor of Philosophy.

LeHNEIS, H. R. \& Wilson, R. G., Jr. (1972). An electric arm orthosis. Bulletin of Prosthetics Research, Department of Medicine and Surgery, Veterans Administration, Washington, D.C.

Nickel, V. L., KarchaK, A., Jr. \& Allen, J. R. (1969). Electrically powered orthotic systems. The fournal of Bone and foint Surgery, Vol. 5 I A, No. 2. 